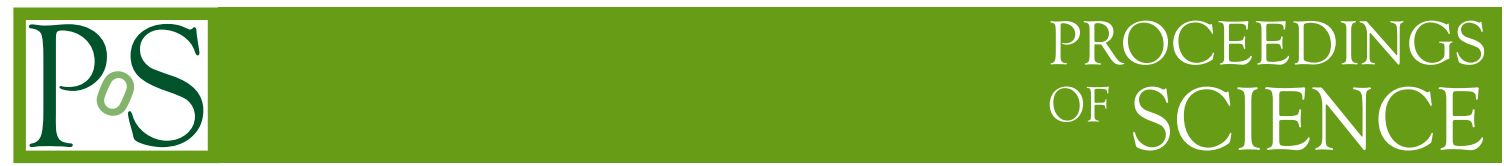

\title{
EW SUSY Production at the LHC
}

\author{
Holly Pacey ${ }^{a, *}$ on behalf of the ATLAS and CMS Collaborations \\ ${ }^{a}$ Cavendish Laboratory, University of Cambridge \\ JJ Thompson Avenue, Cambridge, UK
}

E-mail: holly.ann.pacey@cern.ch

The direct production of electroweak SUSY particles, including sleptons, charginos, and neutralinos, is a particularly interesting area of search at the LHC. While the lightest neutralino is a well-motivated and studied candidate for dark matter in models with R-parity conservation, the small cross sections of electroweak production leads to difficult searches. This talk will highlight the most recent results of searches performed by the ATLAS and CMS experiments for supersymmetric particles produced via electroweak processes, including analyses with leptonic and hadronic final states.

*** Particles and Nuclei International Conference - PANIC2021 ***

*** 5 - 10 September, $2021 * * *$

$* * *$ Online $* * *$

${ }^{*}$ Speaker 
Whilst there are many motivations to search for Supersymmetry (SUSY) in general, electroweak supersymmetry has unique motivations. In addition to being currently less constrained than strong SUSY production, light Higgsinos are favoured by naturalness arguments and light gauginos and sleptons are favoured by the dark matter relic density [1]. Sleptons and neutralinos also affect the muon $g_{\mu}-2$ value and, for masses between 0.2 and $1.0 \mathrm{TeV}$, they can lead to a $g_{\mu}-2$ value consistent with the recently observed anomaly [2]. The searches considered in this talk were interpreted in models consistent with these motivations. First, Gauge-Mediated SUSY breaking (GMSB) models are considered - with gravitino or goldstino lightest-SUSY-particles (LSPs) and Higgsino nextlightest-SUSY-particles (NLSPs). Second, simplified models are considered, where wino and bino gauginos are in the MSSM parameter space consistent with the $g_{\mu}-2$ anomaly.

This talk presented the latest results from the ATLAS [3] and CMS [4] experiments: four full Run 2 searches for gaugino pair-production decaying to a variety of final states. The pair-produced gauginos are the NLSPs for each interpretation, and decay into either $W, Z$ or Higgs bosons and the LSP, which leaves a decay signature including missing transverse momentum $\left(p_{\mathrm{T}}^{\text {miss }}\right)$. These searches use $p_{\mathrm{T}}^{\text {miss }}$ triggers, allowing looser requirements on the other objects in the final state. The dominant uncertainties in all of the searches come from Monte Carlo (MC) statistics.

The first two searches discussed are the first SUSY searches with a fully-hadronic final state, at ATLAS [5] and CMS [6]. Following this an ATLAS search with a three-lepton final state is presented [7], and finally a four- $b$-jet final state search from CMS [8].

The ATLAS fully-hadronic search [5] has a signature of two boosted bosons each decaying to a large-radius jet and $p_{\mathrm{T}}^{\text {miss }}$, following the diagrams shown in Figure 1. The search has extensive interpretations, for example the MSSM — considering many options of the NLSP and LSP identities — and a GMSB model.

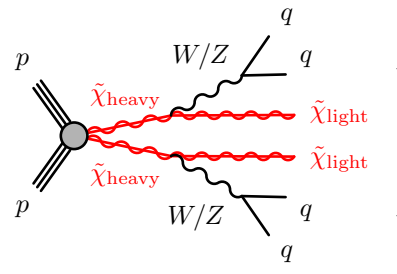

(a)

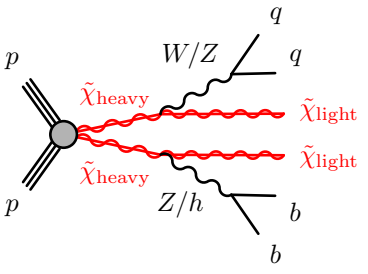

(b)

Figure 1: Feynman diagrams for the fully-hadronic ATLAS search [5].

The basis for sensitivity in this search comes from the use of boosted $W / Z \rightarrow q q$ taggers, which were specially re-optimised for this case. The taggers are based on a neural network using the large-radius jet mass, the track multiplicity in the large-radius jet, and the "energy correlation function" that targets 2-prong jets $[9,10]$. The tagger has excellent performance, managing to remove up to $93 \%$ of the background whilst retaining $50 \%$ of the signal.

As with the other searches considered in this talk, the search results are obtained by a simultaneous fit to several orthogonal signal region bins, to maximise sensitivity. The signal regions are split into those with or without any b-jets. The main Standard Model (SM) backgrounds in the search include $Z+$ jets, $W+$ jets and diboson processes, which are estimated using a semi-data-driven approach. For each of these backgrounds, an orthogonal control region is defined, enriched in the 
desired process. A normalisation factor is derived to scale the MC simulation to data in the control region, which is applied to the $\mathrm{MC}$ in the signal regions.

The post-fit agreement between the data and SM background estimate shows consistency with the SM, hence exclusion limits are placed on the many SUSY interpretations considered. In particular, Figure 2a shows limits for the MSSM interpretation for a range of branching ratios for the NLSP to $Z$ boson decay $(\mathcal{B}(N L S P \rightarrow Z)$ ), which has a very small impact on the sensitivity. Figure $2 \mathrm{~b}$ shows instead the GMSB interpretation in the plane of the NLSP mass and $\mathcal{B}$ (NLSP $\rightarrow$ $Z)$. Here the limits complement previous ATLAS results for different final states well.

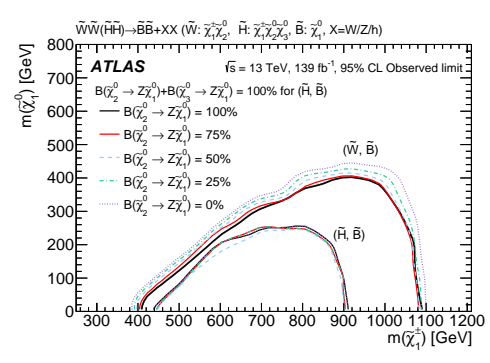

(a) Simplified model

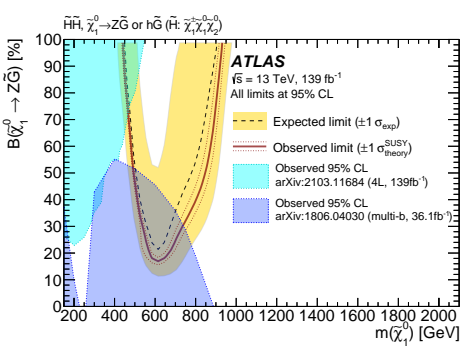

(b) GMSB

Figure 2: Exclusion limits for the fully-hadronic ATLAS search [5].

Across the ring at CMS, a search was performed with the same fully-hadronic signature as ATLAS [6]. As in Figure 1, the $W W, W Z$ or $W h$ mediated decays are considered, with the $Z$ or $h$ decaying to $b$-jets, such that the final state includes two large-radius jets. This search is interpreted in a simplified model with degenerate wino NLSPs and a bino LSP. Like the ATLAS search, this search relies on boosted boson decay taggers, which have a similar performance to that of ATLAS. Unlike ATLAS, CMS has the ability to identify two sub-b-jets within one large-radius jet, leading to three separate taggers: $h / Z \rightarrow b \bar{b}, W \rightarrow q q$ and $W / Z \rightarrow q q$. The signal regions are split into those with or without b-jets, each binned in $p_{\mathrm{T}}^{\text {miss }}$. The main SM backgrounds are again estimated using control regions.

No significant sign of new physics is observed in the post-fit agreement between the data and the SM background estimate, so exclusion limits are placed. The $W W$-mediated decay interpretation is shown in Figure 3a, where a small data deficit in the $0 \mathrm{~b}$-jets signal region strengthens the observed limit. The $W Z$-mediated decay limit is shown in Figure 3b. The limit for the $W h$-mediated decay interpretation is shown in Figure 3c, where a small data excess weakens the observed limit. These results can be compared to those from ATLAS, with the same simplified model interpretation. The $W Z$ and $W h$ sensitivity is very similar, whereas the $W W$ sensitivity from ATLAS shows very good complementarity to the CMS result.

The leptonic ATLAS search [7] considers the same $W Z$ or $W Z$ mediated decays as in Figure 1a, but with the bosons decaying leptonically. This search has two MSSM interpretations: firstly the same model as the CMS hadronic search, and secondly a Higgsino model, with the NLSP and LSP having very similar masses. Three orthogonal search regions - ' $W h$ ', 'on-shell $W Z$ ' and 'off-shell $W Z$ ' - are considered. The 'off-shell $W Z$ ' region targets the Higgsino model by selecting the compressed mass region. Here the small Higgsino mass splitting substantially increases the chance of the $W-$ or $Z$-boson being produced off-shell. In this region the fake-lepton background is 


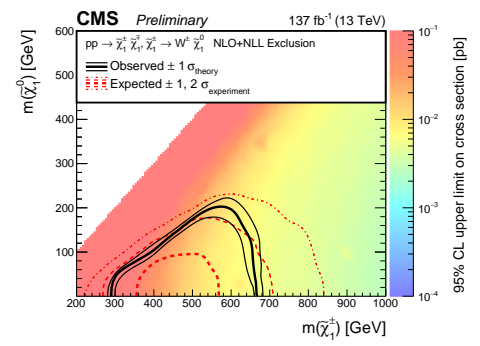

(a) $W W$

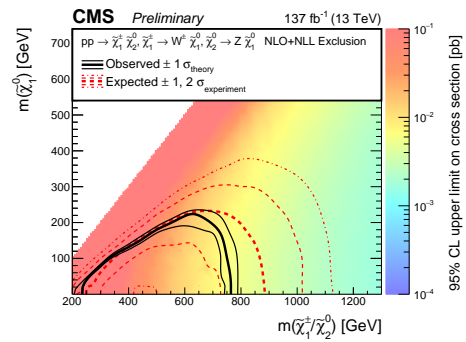

(b) $W Z$

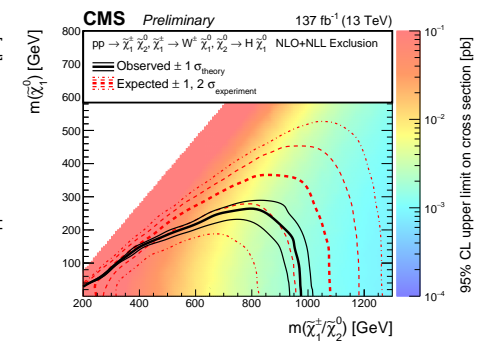

(c) $W h$

Figure 3: Exclusion limits for the fully-hadronic CMS search [6].

prominent; it is identified and removed with a 70-90\% signal efficiency using a BDT-based tagger.

The 'off-shell $W Z$ ' region is split into high and low $p_{\mathrm{T}}^{\text {miss }}$, along with 0 or $>0$ jets, to allow for ISR (initial state radiation) activity. The 'on-shell $W Z$ ' region is binned in $p_{\mathrm{T}}^{\text {miss }}$ and transverse mass, The main SM background in this search is $W Z$, estimated using the control region approach.

The post-fit agreement between the data and SM background estimate shows no significant sign of new physics, and so exclusion limits can be placed in the NSLP-LSP mass plane. For the $W Z$ interpretation, the signal regions in this search are combined with those from a previous search for the compressed-mass region with a two-lepton final state [11], leading to excellent sensitivity in the limits shown in Figures $4 a$ and 4b. For the Wh interpretation, limits are shown in Figure 4c, where the observed limit is slightly weakened due to small data excesses in the $W h$ signal regions. The $W Z$ limits are comparable to the most recent CMS search for the same final state [12], whereas the CMS $W h$ limits are slightly stronger than ATLAS's.

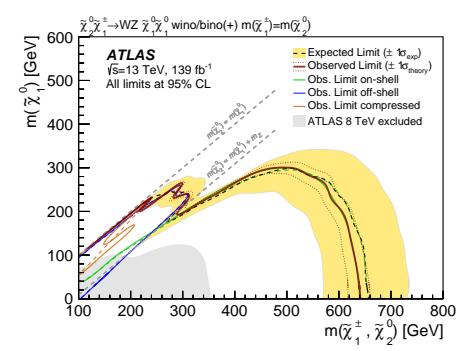

(a) 'off-shell WZ')

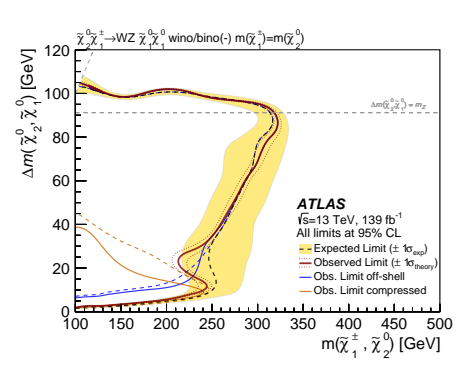

(b) 'on-shell WZ'

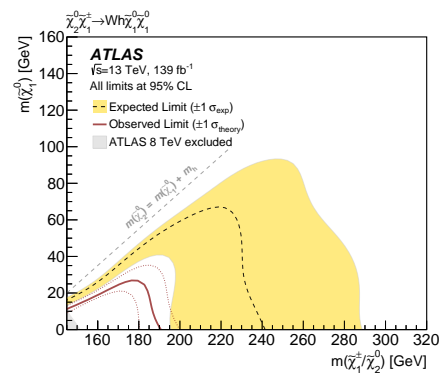

(c) 'Wh'

Figure 4: Exclusion limits for the three-lepton ATLAS search [7].

The CMS 4-b search [8] considers Higgs mediated neutralino decays, where each Higgs boson decays to two b-jets. The search has two interpretations: either a GMSB model, or a simplified model. Two cases are considered; the first is a resolved channel where the final state jets are small-radius. The second is a boosted scenario, where each Higgs decays to one large-radius jet, to provide sensitivity to more compressed mass space. Crucially for this search is the quality of b-jet tagging, which for CMS can either be done for small- or large-radius jets. For the large-radius case this provides a $90 \%$ efficiency to identify $h \rightarrow b b$ candidates.

Both sets of signal regions are binned in $p_{\mathrm{T}}^{\text {miss }}$. The background is estimated using a data-driven $\mathrm{ABCD}$ approach, where the background estimate in the signal region is obtained by scaling the 
data in a signal-free orthogonal sideband region. The scaling is derived as the ratio between data in regions similar to the signal and sideband, but with a lower b-jet multiplicity. The post-fit agreement between the data and SM background estimate contains one locally-significant excess in data in bin 11 , with a global significance of only $1.9 \sigma$. As such, exclusion limits can once again be placed, although the observed excess weakens the observed exclusion sensitivity relative to that expected. The GMSB interpretation is given as the upper limit on the cross-section as a function of NLSP mass, excluding Higgsino masses between $175-1025 \mathrm{GeV}$. In comparison, the most recent ATLAS search in this final state, using the partial Run-2 dataset, excluded $290-890 \mathrm{GeV}$.

To summarise, these latest searches comprehensively expand the excluded gaugino phase space even further. Constraints on gauginos are approaching the $\mathrm{TeV}$ scale, however there is still more well-motivated phase space to explore. For example, moving to slepton production, there are many models consistent with the g-2 anomaly that are yet to be probed [13] and can inform the direction of future searches for electroweak SUSY at the LHC.

\section{References}

[1] J. Hisano, S. Matsumoto, M. Nagai, O. Saito and M. Senami, Non-perturbative effect on thermal relic abundance of dark matter, Phys. Lett. B 646 (2007) 34 [arXiv: 0610249].

[2] Muon $g$ - 2 Collaboration collaboration, Measurement of the Positive Muon Anomalous Magnetic Moment to 0.46 ppm, Phys. Rev. Lett. 126 (2021) 141801.

[3] ATLAS Collaboration, The ATLAS Experiment at the CERN Large Hadron Collider, JINST 3 (2008) S08003.

[4] CMS Collaboration, The CMS experiment at the CERN LHC, JINST 3 (2008) S08004.

[5] ATLAS Collaboration, Search for charginos and neutralinos in final states with two boosted hadronically decaying bosons and missing transverse momentum in p collisions at $\sqrt{s}=13 \mathrm{TeV}$ with the ATLAS detector, Phys. Rev. D 104 (2021) 112010 [arXiv: 2108.07586].

[6] CMS Collaboration, "Search for electroweak production of supersymmetric particles in final states containing hadronic decays of WW, WZ, or WH and missing transverse momentum." CMS-PAS-SUS-21-002, 2021.

[7] ATLAS Collaboration, Search for chargino-neutralino pair production in final states with three leptons and missing transverse momentum in $\sqrt{s}=13 \mathrm{TeV}$ pp collisions with the ATLAS detector, Eur. Phys. J. C 81 (2021) 1118 [arXiv:2106.01676].

[8] CMS Collaboration, "Search for higgsinos in channels with two Higgs bosons and missing transverse momentum in proton-proton collisions at $\sqrt{s}=13 \mathrm{TeV}$." CMS-PAS-SUS-20-004, 2020.

[9] ATLAS Collaboration, Performance of top-quark and W-boson tagging with ATLAS in Run 2 of the LHC, Eur. Phys. J. C 79 (2019) 375 [arXiv: 1808.07858].

[10] ATLAS Collaboration, "Boosted hadronic vector boson and top quark tagging with ATLAS using Run 2 data." ATL-PHYS-PUB-2020-017, 2020.

[11] ATLAS Collaboration, Searches for electroweak production of supersymmetric particles with compressed mass spectra in $\sqrt{s}=13 \mathrm{TeV}$ pp collisions with the ATLAS detector, Phys. Rev. D 101 (2020) 052005.

[12] CMS Collaboration, Search for electroweak production of charginos and neutralinos in proton-proton collisions at $\sqrt{s}=13 \mathrm{TeV}$, Submitted to JHEP (2021) [arXiv: 2106.14246].

[13] ATLAS Collaboration, “SUSY Summary Plots June 2021.” ATLAS-PHYS-PUB-2021-019, 2021. 